Proceedings of the Edinburgh Mathematical Society (2007) 50, 551-561 (C)

DOI:10.1017/S0013091505001598 Printed in the United Kingdom

\title{
LARGEST 2-GENERATED SUBSEMIGROUPS OF THE SYMMETRIC INVERSE SEMIGROUP
}

\author{
J. M. ANDRÉ ${ }^{1}$, V. H. FERNANDES ${ }^{1}$ AND J. D. MITCHELL ${ }^{2}$ \\ ${ }^{1}$ Centro de Álgebra da Universidade de Lisboa, \\ Avenida Professor Gama Pinto 2, \\ 1649003 Lisboa, Portugal and \\ Departamento de Matemática, Faculdade de Ciências \\ e Tecnologia da Universidade Nova de Lisboa, \\ Monte da Caparica, 2829516 Caparica, Portugal \\ (jmla@fct.unl.pt; vhf@fct.unl.pt) \\ ${ }^{2}$ Mathematical Institute, University of St Andrews, North Haugh, \\ St Andrews, Fife KY16 9SS, UK (jdm3@st-and.ac.uk)
}

(Received 14 November 2005)

\begin{abstract}
The symmetric inverse monoid $\mathcal{I}_{n}$ is the set of all partial permutations of an $n$-element set. The largest possible size of a 2-generated subsemigroup of $\mathcal{I}_{n}$ is determined. Examples of semigroups with these sizes are given. Consequently, if $M(n)$ denotes this maximum, it is shown that $M(n) /\left|\mathcal{I}_{n}\right| \rightarrow 1$ as $n \rightarrow \infty$. Furthermore, we deduce the known fact that $\mathcal{I}_{n}$ embeds as a local submonoid of an inverse 2-generated subsemigroup of $\mathcal{I}_{n+1}$.
\end{abstract}

Keywords: inverse semigroups; generators; subsemigroups

2000 Mathematics subject classification: Primary 20M20

Secondary $20 \mathrm{M} 18$

\section{Introduction and the statements of the main theorems}

The topic of embedding a semigroup into a 2-generated semigroup is classical. Sierpiński $[\mathbf{1 1}]$ and Banach [1] proved that every countable semigroup, being isomorphic to a semigroup of mappings on $\mathbb{N}$, can be embedded in a 2-generated subsemigroup of the monoid of all mappings from $\mathbb{N}$ to $\mathbb{N}$. Evans [2] and Neumann [8] followed with their own proofs, involving presentations and wreath products, respectively. As a consequence of Neumann's proof it follows that any finite semigroup can be embedded in a finite 2-generator semigroup. A more elementary method can be used to prove the same result. If $\mathcal{T}_{n}$ denotes the monoid of all mappings from an $n$-element set to itself, then the semigroup theoretic analogue of Cayley's theorem for groups states that every semigroup with $n-1$ elements embeds in a subsemigroup of $\mathcal{T}_{n}$. In [7] it is shown that $\mathcal{T}_{n}$ embeds in a 2-generator subsemigroup of $\mathcal{T}_{n+1}$. Thus, Neumann's result is obtained. 
The topic of this paper is, however, not semigroups in general but a special class of semigroups called inverse semigroups. Ash $[\mathbf{3}]$ proved that every countable inverse semigroup $S$ can be embedded in a 4-generator inverse semigroup $T$, that is, a 4-generator subsemigroup that happens to be an inverse semigroup itself. A partial permutation of a set $X$ is just an injective mapping with domain contained in or equal to $X$. Ash's result can be obtained by proving that any countable collection of partial permutations on $\mathbb{N}$ can be generated by two such partial permutations and their inverses; see [4, Proposition 4.2]. It is also shown in [3] that if $S$ happens to be finite, then $S$ embeds in a finite $T$. A different proof of this is given in [7]. Again analogous to Cayley's theorem, every inverse semigroup embeds in the symmetric inverse monoid $\mathcal{I}_{n}$, the monoid of all partial permutations of an $n$-element set. The result then follows from the fact that $\mathcal{I}_{n}$ embeds in a 2-generator inverse subsemigroup of $\mathcal{I}_{n+2}[\mathbf{7}]$.

Recently, Holzer and König [5] attempted to answer the question: what is the largest possible size of a 2-generated subsemigroup of $\mathcal{T}_{n}$ ? Their paper connects the standard study of 2-generated semigroups to theoretical computer science. Amongst other things, Holzer and König show that when $n$ is prime the largest 2-generated subsemigroup of $\mathcal{T}_{n}$ lies in a class of explicitly defined semigroups. The precise semigroup in this class, with largest size, is, as yet, unknown except for small values of $n$. Answering the question when $n$ is not a prime seems to be a rather difficult problem. After attempting to find such an answer, without success, we followed Pólya's advice [10], and considered a seemingly more straightforward question. The outcome of this consideration is the topic of this paper. The intention is to prove the following theorems.

Theorem 1.1. If $n \geqslant 10$ is even, then the largest size of a 2-generated subsemigroup of $\mathcal{I}_{n}$ is

$\mathfrak{e}(n)=\varepsilon(n)+\frac{1}{36}\left(n^{6}+3 n^{5}+13 n^{4}-411 n^{3}+1390 n^{2}-1320 n+36\right)(n-3) !+\sum_{r=0}^{n-4}\left(\begin{array}{l}n \\ r\end{array}\right)^{2} r !$,

where $\varepsilon(n)=3(n-3)$, if $3 \nmid n$, and $\varepsilon(n)=2(n-3)$, if $3 \mid n$. Moreover, there are inverse subsemigroups of $\mathcal{I}_{n}$ generated by two elements with size $\mathfrak{e}(n)$.

Theorem 1.2. If $n \geqslant 7$ is odd, then the largest size of a 2-generated subsemigroup of $\mathcal{I}_{n}$ is

$$
\mathfrak{o}(n)=2 n-4+\frac{1}{4}\left(n^{4}+2 n^{3}-23 n^{2}+36 n-12\right)(n-2) !+\sum_{r=0}^{n-3}\left(\begin{array}{l}
n \\
r
\end{array}\right)^{2} r ! .
$$

Moreover, there are inverse subsemigroups of $\mathcal{I}_{n}$ generated by two elements with size $\mathfrak{o}(n)$.

These theorems are proved in $\S \S 3$ and 4 . The cases when $n<10$ is even and when $n<7$ is odd are considered in $\S 5$. The semigroup $\mathcal{I}_{n}$ is itself 2 -generated when $n<3$. A corollary of the construction, in $\S 4$, of subsemigroups with sizes $\mathfrak{o}(n)$ and $\mathfrak{e}(n)$, is a slight improvement of the main theorem of $[\mathbf{7}]$. That is, $\mathcal{I}_{n}$ can be embedded, as a local submonoid, in an inverse 2-generated subsemigroup of $\mathcal{I}_{n+1}$. It is stated in the acknowledgements of $[\mathbf{7}]$ that this result was obtained by the referee of the paper. For 
undefined terms in, and further information about, semigroup theory, the reader should consult $[6]$.

\section{Preliminaries}

Before beginning the proofs of Theorems 1.1 and 1.2, a few observations and definitions are required. If $X$ is a subset of a semigroup $S$, then denote by $\langle X\rangle$ the subsemigroup generated by $X$, that is, the semigroup where every element can be given as a product of elements from $X$. The domain of $\alpha \in \mathcal{I}_{n}$ is the set $\operatorname{dom}(\alpha)=\{x: x \alpha$ is defined $\}$ and the image of $\alpha \in \mathcal{I}_{n}$ is the set $\operatorname{im}(\alpha)=\{x \alpha: x \in \operatorname{dom}(\alpha)\}$. The rank of $\alpha$ is simply the size of its image, denoted by $\operatorname{rank}(\alpha)$. If $\alpha$ is a permutation of its image, then $\langle\alpha\rangle$ is a cyclic group. Thus, it is possible to refer to the order of $\alpha$, which is denoted by $|\alpha|$.

There are $\left(\begin{array}{l}n \\ r\end{array}\right)$ possible domains and $\left(\begin{array}{l}n \\ r\end{array}\right)$ possible images of elements in $\mathcal{I}_{n}$ with rank $r$. Moreover, there are $r$ ! partial permutations with a fixed image and kernel of rank $r$. It follows that the number of elements of rank $r$ in $\mathcal{I}_{n}$ is $\left(\begin{array}{l}n \\ r\end{array}\right)^{2} r$ !. Summing over all $r$ gives

$$
\left|\mathcal{I}_{n}\right|=\sum_{r=0}^{n}\left(\begin{array}{l}
n \\
r
\end{array}\right)^{2} r !
$$

The same line of thought can be used to find an upper bound for the size of any subsemigroup $U$ of $\mathcal{I}_{n}$. If the elements with rank $r$ in $U$ admit $d(r)$ distinct domains and $i(r)$ distinct images, then, as above, there are at most $d(r) i(r) r$ ! elements with rank $r$ in $U$. So, summing over all $r$ yields

$$
|U| \leqslant \sum_{r=0}^{n} d(r) i(r) r !
$$

The forms of $\mathfrak{e}(n)$ and $\mathfrak{o}(n)$ given in Theorems 1.1 and 1.2 arose as simplifications of the slightly longer expressions:

$$
\begin{aligned}
\mathfrak{e}(n)= & \varepsilon(n)+(n-3)^{2}(n-1) ! \\
+ & {\left[\left(\begin{array}{l}
n \\
2
\end{array}\right)-3\right]^{2}(n-2) !+\left[\left(\begin{array}{l}
n \\
3
\end{array}\right)-1\right]^{2}(n-3) !+\sum_{r=0}^{n-4}\left(\begin{array}{l}
n \\
r
\end{array}\right)^{2} r ! }
\end{aligned}
$$

and

$$
\mathfrak{o}(n)=2 n-4+(n-2)^{2}(n-1) !+\left[\left(\begin{array}{l}
n \\
2
\end{array}\right)-1\right]^{2}(n-2) !+\sum_{r=0}^{n-3}\left(\begin{array}{l}
n \\
r
\end{array}\right)^{2} r !
$$

These lengthier versions of $\mathfrak{e}(n)$ and $\mathfrak{o}(n)$ also make their relationship with $\left|\mathcal{I}_{n}\right|$ more apparent.

\section{Not larger than $\mathfrak{e}(n)$ or $\mathfrak{o}(n)$}

In this section, we prove that any 2-generated subsemigroup of $\mathcal{I}_{n}$ has size at most $\mathfrak{e}(n)$ in the even case and at most $\mathfrak{o}(n)$ in the odd case. At several points in this section, an 
upper bound on the order of any element of the symmetric group of degree $m \leqslant n$ is required. The largest order of an element in $\mathcal{S}_{m}$ is known as Landau's function $\lambda(m)$, and it is the greatest least common divisor of any partition of $m$. Several tight bounds are known for Landau's function. However, for our purposes it will suffice to note that, if $m \geqslant 4$, and $\alpha \in \mathcal{S}_{m}$, then by induction on $m$ we obtain

$$
|\alpha| \leqslant(m-1) !
$$

Let us begin in earnest by proving that any pair of non-permutations in $\mathcal{I}_{n}$ generates a semigroup with size less than $\mathfrak{e}(n)$.

Lemma 3.1. If $\alpha, \beta \in \mathcal{I}_{n} \backslash \mathcal{S}_{n}$ and $n \geqslant 5$, then $|\langle\alpha, \beta\rangle| \leqslant \mathfrak{e}(n)<\mathfrak{o}(n)$.

Proof. By (2.2) and (2.3),

$$
\mathfrak{o}(n)-\mathfrak{e}(n) \geqslant \frac{1}{3}(n-3) !\left(13 n^{3}-54 n^{2}+47 n+15\right)-n+5 \geqslant(n-3) !-n+5>0,
$$

when $n \geqslant 5$. Therefore, $\mathfrak{e}(n)<\mathfrak{o}(n)$ for all $n \geqslant 5$.

If $a$ and $b$ are elements missing from the images of $\alpha$ and $\beta$, then any element in $\langle\alpha, \beta\rangle$ is missing either $a$ or $b$ from its image. Likewise, if $c \notin \operatorname{dom}(\alpha)$ and $d \notin \operatorname{dom}(\beta)$, then either $c \notin \operatorname{dom}(\mu)$ or $d \notin \operatorname{dom}(\mu)$ for all $\mu \in\langle\alpha, \beta\rangle$. Thus, it is not possible to choose all the elements missing from $\operatorname{im}(\mu)$ or $\operatorname{dom}(\mu)$ from the complement of $\{a, b\}$ or $\{c, d\}$, respectively. It follows that the number of distinct domains, and images, that elements of $\langle\alpha, \beta\rangle$ with rank $r$ admit is at most

$$
\left(\begin{array}{l}
n \\
r
\end{array}\right)-\left(\begin{array}{l}
n-2 \\
r-2
\end{array}\right)
$$

Inequality (2.1) tells us that

$$
|\langle\alpha, \beta\rangle| \leqslant \sum_{r=0}^{n-1}\left[\left(\begin{array}{l}
n \\
r
\end{array}\right)-\left(\begin{array}{l}
n-2 \\
r-2
\end{array}\right)\right]^{2} r ! .
$$

Now, the proof is completed by showing that the coefficients of each of the terms $r$ ! in (3.2) are not greater than the corresponding coefficients in $(2.2)$. When $r=0,1, \ldots, n-4$ this is obvious. Simplify the remaining terms in (3.2) to obtain

$$
4(n-1) !+\left[\left(\begin{array}{l}
n \\
2
\end{array}\right)-\left(\begin{array}{c}
n-2 \\
2
\end{array}\right)\right]^{2}(n-2) !+\left[\left(\begin{array}{l}
n \\
3
\end{array}\right)-\left(\begin{array}{c}
n-2 \\
3
\end{array}\right)\right]^{2}(n-3) !
$$

Comparing these coefficients with those in $(2.2), 4 \leqslant(n-3)^{2},\left(\begin{array}{c}n-2 \\ 2\end{array}\right) \geqslant 3$ and $\left(\begin{array}{c}n-2 \\ 3\end{array}\right) \geqslant 1$ when $n \geqslant 5$, and the result follows.

If $\alpha, \beta \in \mathcal{S}_{n}$, then $|\langle\alpha, \beta\rangle| \leqslant n !<\mathfrak{e}(n)$ when $n \geqslant 4$. Therefore, it remains to prove that any permutation together with any non-permutation in $\mathcal{I}_{n}$ generate a subsemigroup with size less than $\mathfrak{e}(n)$ in the even case and less than $\mathfrak{o}(n)$ in the odd case. The next simple lemma is used in the proof of both cases. Denote by $\alpha_{i}$ the cycle of $\alpha \in \mathcal{S}_{n}$ containing the number $i$. 
Lemma 3.2. If $\alpha \in \mathcal{S}_{n}$ and $\beta \in \mathcal{I}_{n} \backslash \mathcal{S}_{n}$ with $a \notin \operatorname{dom}(\beta)$ and $b \notin \operatorname{im}(\beta)$, then

$$
|\langle\alpha, \beta\rangle| \leqslant|\alpha|+\sum_{r=0}^{s}\left[\left(\begin{array}{l}
n \\
r
\end{array}\right)-\left(\begin{array}{c}
n-t \\
n-r
\end{array}\right)\right]^{2} r !
$$

where $s=\operatorname{rank}(\beta)$ and $t=\max \left\{\left|\alpha_{a}\right|,\left|\alpha_{b}\right|\right\}$.

Proof. Any element $\mu \neq \alpha^{i}$, for any $i$, of $\langle\alpha, \beta\rangle$ can be written as $\alpha^{i} \beta \omega \beta \alpha^{j}$, or $\alpha^{i} \beta \alpha^{j}$, for some $i, j$ and $\omega \in\langle\alpha, \beta\rangle$. Thus, $a \alpha^{-i} \notin \operatorname{dom}(\mu)$ and $b \alpha^{j} \notin \operatorname{im}(\mu)$. In other words, there is an element in $\alpha_{a}$ that is not in $\operatorname{dom}(\mu)$ and an element in $\alpha_{b}$ that is not in $\operatorname{im}(\mu)$. So, as in the proof of Lemma 3.1, the number of distinct domains that elements of $\langle\alpha, \beta\rangle$ with rank $r$ admit is at most

$$
\left(\begin{array}{l}
n \\
r
\end{array}\right)-\left(\begin{array}{c}
n-\left|\alpha_{a}\right| \\
n-r
\end{array}\right) \leqslant\left(\begin{array}{l}
n \\
r
\end{array}\right)-\left(\begin{array}{c}
n-t \\
n-r
\end{array}\right)
$$

Likewise, the number of distinct images that elements of $\langle\alpha, \beta\rangle$ with rank $r$ admit is at most

$$
\left(\begin{array}{l}
n \\
r
\end{array}\right)-\left(\begin{array}{c}
n-\left|\alpha_{b}\right| \\
n-r
\end{array}\right) \leqslant\left(\begin{array}{l}
n \\
r
\end{array}\right)-\left(\begin{array}{c}
n-t \\
n-r
\end{array}\right)
$$

The inequality in the lemma now follows from (2.1) and the fact that, for all $\mu \in\langle\alpha, \beta\rangle$, $\operatorname{rank}(\mu) \leqslant s$ or $\operatorname{rank}(\mu)=n$.

Using Lemma 3.2 it is now possible to prove the main result of this section in the case where $n$ is even.

Lemma 3.3. If $n \geqslant 10$ is even, $\alpha \in \mathcal{S}_{n}$ and $\beta \in \mathcal{I}_{n} \backslash \mathcal{S}_{n}$, then $|\langle\alpha, \beta\rangle| \leqslant \mathfrak{e}(n)$.

Proof. Let $a \notin \operatorname{dom}(\beta)$ and $b \notin \operatorname{im}(\beta)$. Assume without loss of generality that $\left|\alpha_{a}\right| \leqslant$ $\left|\alpha_{b}\right|$. If $\left|\alpha_{b}\right|=n-3$, then the inequality $|\langle\alpha, \beta\rangle| \leqslant \mathfrak{e}(n)$ follows directly from Lemma 3.2. When $\left|\alpha_{b}\right| \leqslant n-4$, it suffices to prove that

$$
|\alpha|+\sum_{r=n-3}^{n-1}\left[\left(\begin{array}{l}
n \\
r
\end{array}\right)-\left(\begin{array}{c}
4 \\
n-r
\end{array}\right)\right]^{2} r !<\varepsilon(n)+\sum_{r=n-3}^{n-1}\left[\left(\begin{array}{l}
n \\
r
\end{array}\right)-\left(\begin{array}{c}
3 \\
n-r
\end{array}\right)\right]^{2} r !
$$

This is equivalent to proving that

$$
(n-1) !=(n-1)(n-2)(n-3) ! \leqslant \varepsilon(n)+\left(6 n^{3}-25 n^{2}+6 n+25\right)(n-3) !,
$$

since $|\alpha| \leqslant(n-1)$ ! by (3.1). To prove the second inequality it is sufficient to show that $(n-1)(n-2)<6 n^{3}-25 n^{2}+6 n+25$ for $n \geqslant 10$, since $\varepsilon(n)>0$ when $n \geqslant 4$. It is possible to do this using elementary calculus. Indeed, take the real-valued functions $f(x)=x^{2}-3 x+2=(x-1)(x-2)$ and $g(x)=6 x^{3}-25 x^{2}+6 x+25$. Then $f(10)=72<$ $3585=g(10)$. Moreover, if $x \geqslant 3$, then $f^{\prime}(x)<2 x<2 x(9 x-25)<18 x^{2}-50 x+6=g^{\prime}(x)$.

It remains to consider what happens when $\left|\alpha_{b}\right|=n-2, n-1$ or $n$. Note that in this case, since $n$ is even, $|\alpha| \leqslant n$. If $N$ is the number of elements of $\langle\alpha, \beta\rangle$ of $\operatorname{rank} n-1$, we prove that

$$
N \leqslant|\alpha|^{2}(n-2) ! \leqslant n^{2}(n-2) ! .
$$


If $\operatorname{rank}(\beta)<n-1$, then there are no elements of rank $n-1$ and (3.3) is satisfied. Assume that $\operatorname{rank}(\beta)=n-1$. There are two cases to consider.

First, if $b \alpha^{i} \neq a$, for all $i$, then any product of $\alpha \mathrm{s}$ and $\beta \mathrm{s}$, containing more than 1 occurrence of $\beta$, has rank at most $n-2$. Consequently, there are at most $|\alpha|^{2} \leqslant n^{2}$ elements of rank $n-1$.

Second, if there exists $i \in \mathbb{Z}$ such that $b \alpha^{i}=a$, then $\operatorname{dom}\left(\alpha^{i} \beta\right)=\operatorname{im}\left(\alpha^{i} \beta\right)$ and the unique element not in this set is $b$. Note that since $\alpha^{i} \beta$ is a permutation of its domain, which has size $n-1,\left|\alpha^{i} \beta\right| \leqslant(n-2)$ ! by (3.1). As in the previous case, we will prove that every element of $\langle\alpha, \beta\rangle$ with rank $n-1$ has the form $\alpha^{j}\left(\alpha^{i} \beta\right)^{k} \alpha^{l}$ for some $j, k, l$. To this end observe that if $x \alpha^{k}=x$, for some $k$ and some $x$ in $\alpha_{b}$, then $y \alpha^{k}=y$ for all $y$ in $\alpha_{b}$. Moreover, since $\left|\alpha_{b}\right|=n-2, n-1$ or $n$, and $n$ is even, it follows that $\alpha^{k}$ is the identity permutation $1_{n}$. Taking the contrapositive, if $\alpha^{k} \neq 1_{n}$, then $y \alpha^{k} \neq y$ for all $y$ in $\alpha_{b}$. In particular, $b \alpha^{k} \neq b$. Therefore, every element of the form $\omega_{1}\left(\alpha^{i} \beta\right) \alpha^{k}\left(\alpha^{i} \beta\right) \omega_{2}$, $\omega_{1}, \omega_{2} \in\langle\alpha, \beta\rangle$ and $\alpha^{k} \neq 1_{n}$, has rank at most $n-2$. It follows from this that if $\beta \alpha^{k} \beta$ is a factor of an element in $\left\langle\alpha, \alpha^{i} \beta\right\rangle=\langle\alpha, \beta\rangle$ with rank $n-1$, then $k=i$. Thus, any element of rank $n-1$ has the form $\alpha^{j}\left(\alpha^{i} \beta\right)^{k} \alpha^{l}$ and there are at most $|\alpha|^{2}\left|\alpha^{i} \beta\right| \leqslant n^{2}(n-2)$ ! elements of this type. Hence,

$$
h(n)=n+n^{2}(n-2) !+\sum_{r=0}^{n-2}\left(\begin{array}{l}
n \\
r
\end{array}\right)^{2} r ! \geqslant \mid\langle\alpha, \beta\rangle .
$$

To complete the proof we show that

$$
\mathfrak{e}(n)-h(n)=\varepsilon(n)-n+\left(n^{4}-\frac{40}{3} n^{3}+41 n^{2}-\frac{110}{3} n+1\right)(n-3) !>0,
$$

when $n \geqslant 10$.

Now, $\varepsilon(n)-n>n-6>0$ when $n \geqslant 7$ and so it suffices to prove that

$$
n^{4}-\frac{40}{3} n^{3}+41 n^{2}-\frac{110}{3} n+1>0
$$

when $n \geqslant 10$. As above, take the real-valued function

$$
k(x)=x^{4}-\frac{40}{3} x^{3}+41 x^{2}-\frac{110}{3} x+1 .
$$

Then $k(10)=401$ and

$$
k^{\prime}(x)=4 x^{3}-40 x^{2}+82 x-\frac{110}{3}>4 x^{3}-40 x^{2}+80 x-40=4 x\left(x^{2}-10 x+20\right)-40 .
$$

Now, $x(x-10) \geqslant 0>-19$ when $x \geqslant 10$. Thus, $x^{2}-10 x+20>1$ and so $k^{\prime}(x)>0$ when $x \geqslant 10$.

Finally, and again using Lemma 3.2, it is possible to prove the main result in the case that $n$ is odd.

Lemma 3.4. If $n \geqslant 7$ is odd, $\alpha \in \mathcal{S}_{n}$ and $\beta \in \mathcal{I}_{n} \backslash \mathcal{S}_{n}$, then $|\langle\alpha, \beta\rangle| \leqslant \mathfrak{o}(n)$. 
Proof. Let $a \notin \operatorname{dom}(\beta)$ and $b \notin \operatorname{im}(\beta)$. Assume without loss of generality that $\left|\alpha_{a}\right| \leqslant$ $\left|\alpha_{b}\right|$. If $\left|\alpha_{b}\right|=n-2$, then the inequality $|\langle\alpha, \beta\rangle| \leqslant \mathfrak{o}(n)$ follows directly from Lemma 3.2. If $\left|\alpha_{b}\right| \leqslant n-3$, then, by Lemma 3.2, it suffices to prove that

$$
|\alpha|+\sum_{r=n-2}^{n-1}\left[\left(\begin{array}{l}
n \\
r
\end{array}\right)-\left(\begin{array}{c}
3 \\
n-r
\end{array}\right)\right]^{2} r !<2 n-4+\frac{1}{4}\left(n^{4}+2 n^{3}-23 n^{2}+36 n-12\right)(n-2) !
$$

or, equivalently, to prove that

$$
(n-1) ! \leqslant 2 n-4+\left(4 n^{2}-9 n-3\right)(n-2) !,
$$

since $|\alpha|<(n-1)$ !. When $n \geqslant 3,2 n(2 n-5)>2$ and so $4 n^{2}-9 n-3>n-1$ and the result follows in this case.

Now, assume that the length of $\left|\alpha_{b}\right|$ is $n-1$ or $n$. As in the proof of Lemma 3.3, if $N$ denotes the number of elements of $\langle\alpha, \beta\rangle$ with rank $n-1$, then

$$
N \leqslant|\alpha|^{2}(n-2) ! \leqslant n^{2}(n-2) !
$$

Therefore,

$$
|\langle\alpha, \beta\rangle| \leqslant n+n^{2}(n-2) !+\sum_{r=0}^{n-2}\left(\begin{array}{l}
n \\
r
\end{array}\right)^{2} r !
$$

Now, $2 n-4>n$ when $n \geqslant 5$ and the coefficients of $r$ !, $r \neq n-2$, in the two sums are equal. So, we need only verify that the coefficient of $(n-2) !$ in $\mathfrak{o}(n)$, as shown in Theorem 1.2, is greater than that in the last sum. In other words, we must prove that

$$
\begin{aligned}
\frac{1}{4}\left(n^{4}+2 n^{3}-23 n^{2}+36\right. & -12)-\left[n^{2}+\left(\begin{array}{l}
n \\
2
\end{array}\right)^{2}\right] \\
= & \frac{1}{4}\left(n^{4}+2 n^{3}-23 n^{2}+36 n-12\right)-\frac{1}{4}\left(n^{4}-2 n^{3}+5 n^{2}\right) \\
= & \frac{1}{4}\left(4 n^{3}-28 n^{2}+36 n-12\right)>0 .
\end{aligned}
$$

But $0<4 n(n-6)(n-1)-12$ when $n \geqslant 7$ and $4 n(n-6)(n-1)-12=4 n\left(n^{2}-7 n+6\right)-12<$ $4 n\left(n^{2}-7 n+9\right)-12=4 n^{3}-28 n^{2}+36 n-12$, as required. It follows that $|\langle\alpha, \beta\rangle| \leqslant \mathfrak{o}(n)$ for $n \geqslant 7$.

\section{Realizing $\mathfrak{e}(n)$ and $\mathfrak{o}(n)$}

In this section, we complete the proofs of Theorems 1.1 and 1.2 by proving that there are 2-generated subsemigroups of $\mathcal{I}_{n}$ with size $\mathfrak{e}(n)$ and $\mathfrak{o}(n)$. This necessitates two examples to cover the cases when $n$ is odd and when $n$ is even.

The proof of the following elementary result, reportedly first proved in [9], will be required to prove that our two examples are 2 -generated.

Lemma 4.1. If $n \neq 4$ and $\alpha$ is any nonidentity permutation of degree $n$, or $n=4$ and $\alpha \neq\left(\begin{array}{ll}1 & 2\end{array}\right)\left(\begin{array}{l}3 \\ 4\end{array}\right),\left(\begin{array}{ll}1 & 3\end{array}\right)(24)$ or (1 4)(2 3), then there exists $\beta \in \mathcal{S}_{n}$ such that $\langle\alpha, \beta\rangle=\mathcal{S}_{n}$. 
The first of our examples, $\mathcal{O}(n)$, is defined to be

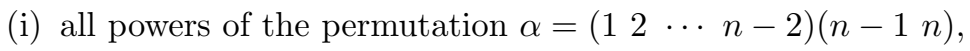

(ii) all elements $\mu \in \mathcal{I}_{n}$, where there exist $d, i \in\{1,2, \ldots, n-2\}$ such that $d \notin \operatorname{dom}(\mu)$ and $i \notin \operatorname{im}(\mu)$.

If $\mu \in \mathcal{O}(n)$, then $\mu^{-1}: x \mu \mapsto x, x \in \operatorname{im}(\mu)$, is the unique inverse of $\mu$ in $\mathcal{I}_{n}$. But there exist $i, d \in\{1,2, \ldots, n-2\}$ such that $d \notin \operatorname{dom}(\mu)=\operatorname{im}\left(\mu^{-1}\right)$ and $i \notin \operatorname{im}(\mu)=\operatorname{dom}\left(\mu^{-1}\right)$. This implies that $\mu^{-1} \in \mathcal{O}(n)$ and so $\mathcal{O}(n)$ is an inverse subsemigroup of $\mathcal{I}_{n}$. The next lemma shows that $\mathcal{O}(n)$ has the desired size and number of generators.

Lemma 4.2. If $n \geqslant 5$ is odd, then $|\mathcal{O}(n)|=\mathfrak{o}(n)$ and $\mathcal{O}(n)$ is 2-generated.

Proof. The first conclusion, that $|\mathcal{O}(n)|=\mathfrak{o}(n)$, follows immediately by (2.3), and since $n$ is odd. Since $n-2$ is odd, $\alpha^{n-2}=(n-1 n)$. Thus, if

$$
\beta=\left(\begin{array}{cccccc}
1 & 2 & 3 & \cdots & n-1 & n \\
- & 3 & 4 & \cdots & n & 2
\end{array}\right),
$$

then together $\alpha^{n-2}$ and $\beta$ generate all permutations on $\{2,3, \ldots, n\}$. So, $\beta^{n-1}=1_{\{2, \ldots, n\}}$, the partial identity with domain $\{2, \ldots, n\}$. If $m=0,1, \ldots, n-3$, then

$$
\alpha^{-m} \beta^{n-1} \alpha^{m}=1_{\{1,2, \ldots, n\} \backslash\{m+1\}}
$$

and so

$$
1_{\{m+2, \ldots, n\}}=\beta^{n-1}\left(\alpha^{-1} \beta^{n-1} \alpha\right)\left(\alpha^{-2} \beta^{n-1} \alpha^{2}\right) \cdots\left(\alpha^{-m} \beta^{n-1} \alpha^{m}\right) .
$$

The partial identity $1_{\{n\}}$ is produced by taking the composition $1_{\{n-1, n\}} \pi 1_{\{n-1, n\}}$ where $\pi \in\left\langle\alpha^{n-2}, \beta\right\rangle$ is the permutation on $\{2,3, \ldots, n\}$ that swaps $n-2$ and $n-1$. Likewise, the empty mapping is produced by taking the composition $1_{\{n\}} \sigma 1_{\{n\}}$, where $\sigma$ is the permutation that swaps $n$ and $n-1$.

Let $\mu \in \mathcal{O}(n)$ be arbitrary with $d, i \in\{1,2, \ldots, n-2\}$ such that $d \notin \operatorname{dom}(\mu)$ and $i \notin \operatorname{im}(\mu)$. If $\operatorname{rank}(\mu)=n$ or 0 , then $\mu$ is a power of $\alpha$ or the empty mapping. Either way $\mu \in\langle\alpha, \beta\rangle$.

Assume that $\operatorname{rank}(\mu)=n-m$ for some $m \in\{1,2, \ldots, n-1\}$. Then $1 \notin \operatorname{dom}(\mu) \alpha^{-d+1}$ and $1 \notin \operatorname{im}(\mu) \alpha^{-i+1}$. It follows that 1 is in neither the domain nor the image of $\alpha^{d-1} \mu \alpha^{-i+1}$. Therefore, there exists a (partial) permutation $\hat{\mu} \in\langle\alpha, \beta\rangle$ of $\{2,3, \ldots, n\}$ such that $\left.\hat{\mu}\right|_{\operatorname{dom}(\mu) \alpha^{-d+1}}=\alpha^{d-1} \mu \alpha^{-i+1}$.

Then let $\nu$ be any permutation of $\{2,3, \ldots, n\}$ such that

$$
\{m+1, \ldots, n\} \nu=\operatorname{dom}(\mu) \alpha^{-d+1} .
$$

Of course, $\nu \in\langle\alpha, \beta\rangle$. With this definition

$$
\alpha^{-d+1} \nu^{-1} 1_{\{m+1, \ldots, n\}} \nu \alpha^{d-1}=\alpha^{-d+1} 1_{\operatorname{dom}(\mu) \alpha^{-d+1}} \alpha^{d-1}=1_{\operatorname{dom}(\mu)} .
$$

So, to conclude, if $x \in \operatorname{dom}(\mu)$, then

$$
(x) 1_{\operatorname{dom}(\mu)} \alpha^{-d+1} \hat{\mu} \alpha^{i-1}=\left(x \alpha^{-d+1}\right) \hat{\mu} \alpha^{i-1}=\left(x \alpha^{-d+1}\right) \alpha^{d-1} \mu \alpha^{-i+1} \alpha^{i-1}=x \mu,
$$

and $1_{\operatorname{dom}(\mu)} \alpha^{-d+1} \hat{\mu} \alpha^{i-1}$ is undefined on the complement of $\operatorname{dom}(\mu)$. Thus, $\mu \in\langle\alpha, \beta\rangle$. 
Table 1. Maximum size of a 2-generated subsemigroup of $\mathcal{I}_{n}, n$ even

\begin{tabular}{rr}
\hline$n$ & $M(n)$ \\
4 & $141^{*}$ \\
6 & $8509^{*}$ \\
8 & $1079625^{*}$ \\
10 & 200798485 \\
12 & 48777044515 \\
14 & 15243109621301 \\
\hline
\end{tabular}

The second of the required semigroups, $\mathcal{E}(n)$, is defined to be

(i) all powers of the permutation $\alpha=(12 \cdots n-3)(n-2 n-1)$, or $(12 \cdots n-$ $3)(n-2 n-1 n)$, when $3 \mid n$ or $3 \nmid n$, respectively,

(ii) all elements $\mu \in \mathcal{I}_{n}$ with $d, i \in\{1,2, \ldots, n-3\}$ satisfying $d \notin \operatorname{dom}(\mu)$ and $i \notin \operatorname{im}(\mu)$.

It is possible to verify that $\mathcal{E}(n)$ is an inverse subsemigroup of $\mathcal{I}_{n}$ in the same way that $\mathcal{O}(n)$ was shown to be.

Lemma 4.3. If $n \geqslant 6$ is even, then $|\mathcal{E}(n)|=\mathfrak{e}(n)$ and $\mathcal{E}(n)$ is 2-generated.

Proof. As in the proof of Lemma 4.2, the first conclusion, that $|\mathcal{E}(n)|=\mathfrak{e}(n)$, follows immediately by (2.2), and since $n$ is even. If $3 \mid n$, then $\alpha^{n-3}=(n-2 n-1)$, and if $3 \nmid n$, then $\alpha^{n-3}=(n-2 n-1 n)$. In either case, Lemma 4.1 guarantees that it is possible to find a permutation $\beta$ of $\{2,3, \ldots, n\}$ such that together $\alpha^{n-3}$ and $\beta$ generate all permutations of $\{2,3, \ldots, n\}$. For example, if $3 \mid n$, then $\beta$ can be

$$
\left(\begin{array}{cccccc}
1 & 2 & 3 & \cdots & n-1 & n \\
- & 3 & 4 & \cdots & n & 2
\end{array}\right)
$$

and if $3 \nmid n$, then $\beta$ can be

$$
\left(\begin{array}{ccccccc}
1 & 2 & 3 & \cdots & n-2 & n-1 & n \\
- & 3 & 4 & \cdots & 2 & n & n-1
\end{array}\right) .
$$

The rest of the proof is, more or less, identical to that of Lemma 4.2 and, for brevity, it is omitted.

\section{Small values, asymptotics and embedding $\mathcal{I}_{n}$ in $\mathcal{I}_{n+1}$}

As the title suggests, in this section some small values of the maximum size $M(n)$ of a 2-generated subsemigroup of $\mathcal{I}_{n}$ are given. When $n \geqslant 7$ and odd, or $n \geqslant 10$ and even, $M(n)$ is precisely $\mathfrak{o}(n)$ or $\mathfrak{e}(n)$, respectively. The asymptotic behaviour of the ratio $M(n) /\left|\mathcal{I}_{n}\right|$ is also studied. The first few values of $M(n)$ are given in Tables 1 and 2 . The values marked with an asterisk were not obtained by applying Theorems 1.1 and 1.2; all 
Table 2. Maximum size of a 2-generated subsemigroup of $\mathcal{I}_{n}, n$ odd

\begin{tabular}{rr}
\hline$n$ & $M(n)$ \\
\hline 3 & $31^{*}$ \\
5 & $934^{*}$ \\
7 & 103692 \\
9 & 15561168 \\
11 & 3180734980 \\
13 & 860918107056 \\
15 & 299336064843732 \\
\hline
\end{tabular}

the other values were. The values when $n=3$ or 4 were obtained by computation. The remaining values, when $n=5,6$, or 8 , were obtained using Lemma 3.1 and arguments analogous to those used in the proof of Lemmas 3.3 and 3.4. The largest 2-generated subsemigroups of $\mathcal{I}_{n}$ in these cases are not always the same as the semigroups $\mathcal{O}(n)$ and $\mathcal{E}(n)$. The following two examples describe 2-generated semigroups with the largest possible size when $n=3,4,5,6$ and 8 .

Example 5.1. If $n=3$, then the partial permutations

$$
\alpha=\left(\begin{array}{lll}
1 & 2 & 3 \\
3 & 1 & 2
\end{array}\right) \quad \text { and } \beta=\left(\begin{array}{ccc}
1 & 2 & 3 \\
- & 1 & 3
\end{array}\right)
$$

generate an inverse subsemigroup of $\mathcal{I}_{n}$ with size 31 . Moreover, this semigroup consists of all partial permutations of $\{1,2,3\}$ with rank at most 2 and the powers of $\alpha$. The semigroup $\mathcal{O}(5)$ has size 934 .

Example 5.2. When $n=4,6$ or 8 , the semigroups with the largest possible size are found by taking a cycle $\alpha$ of order $n$ in $\mathcal{S}_{n}$ together with a group element $\beta$ of rank $n-1$ with maximum possible order, that is, 3,6 or 12 , respectively. The semigroup $\langle\alpha, \beta\rangle$ contains all the elements of rank at most $n-2, n^{2}|\beta|$ elements of rank $n-1$ and the $n$ powers of $\alpha$.

The paper is concluded by making some easy observations.

Lemma 5.3. The sequence $M(n) /\left|\mathcal{I}_{n}\right|$ tends to 1 as $n \rightarrow \infty$.

Proof. The sequence $\mathfrak{o}(n) /\left|\mathcal{I}_{n}\right|$ tends to 1 as $n \rightarrow \infty$. Thus, since $\mathfrak{o}(n) \leqslant \mathfrak{e}(n+1)$, the result follows.

From the definition of the semigroups $\mathcal{O}(n)$ and $\mathcal{E}(n)$, we deduce the following results. As mentioned in $\S 1$, this is already known (see [7]).

Theorem 5.4. The inverse semigroup $\mathcal{I}_{n}, n \geqslant 4$, can be embedded, as a local submonoid, in an inverse 2-generated subsemigroup of $\mathcal{I}_{n+1}$. 
Proof. It is well known that the symmetric inverse monoid on the set $\{2,3, \ldots, n\}$ is generated by the permutations $(23),(23 \cdots n)$ and the idempotent

$$
\left(\begin{array}{cccccc}
1 & 2 & 3 & \cdots & n-1 & n \\
- & - & 3 & \cdots & n-1 & n
\end{array}\right)
$$

(see, for example, [6, Exercise 5.11.6]).

From the definition of $\mathcal{O}(n)$ and $\mathcal{E}(n)$ it is clear that these three partial permutations are elements of both of these monoids.

Acknowledgements. J.M.A. and V.H.F. were partly supported by the FCT and FEDER within the project POCTI-ISFL-1-143 of the Centro de Álgebra da Universidade de Lisboa. J.D.M. acknowledges the support of EPSRC Grant no. GR/S/56085/01.

\section{References}

1. S. Banach, Sur un theorème de M. Sierpiński, Fund. Math. 25 (1935), 5-6.

2. T. Evans, Embedding theorems for multiplicative systems and projective geometries, Proc. Am. Math. Soc. 3 (1952), 614-620.

3. T. E. HALL, Inverse and regular semigroups and amalgamation: a brief survey, in Proc. Symp. on Regular Semigroups, Northern Illinois University, 1979 (Springer, 1979).

4. P. M. Higgins, J. M. Howie, J. D. Mitchell And N. RušKuC, Countable versus uncountable ranks in infinite semigroups of transformations and relations, Proc. Edinb. Math. Soc. 46 (2003), 531-544.

5. M. Holzer AND B. KÖNIG, On deterministic finite automata and syntactic monoid size, Theor. Comp. Sci. 327 (2004), 319-347.

6. J. M. HowIE, Fundamentals of semigroup theory, London Mathematical Society Monographs, New Series, Volume 12 (Oxford University Press, 1995).

7. D. B. McAlister, J. B. Stephen And A. S. Vernitski, Embedding $\mathcal{I}_{n}$ in a 2-generator inverse subsemigroup of $\mathcal{I}_{n+2}$, Proc. Edinb. Math. Soc. 45 (2002), 1-4.

8. B. H. Neumann, Embedding theorems for semigroups, J. Lond. Math. Soc. 35 (1960), 184-192.

9. S. PiCARD, Sur les bases du groupe symetrique et du groupe alternant, Math. Ann. 116 (1939), 752-767.

10. G. Pólya, How to solve it, Princeton Science Library (Princeton University Press, 1988).

11. W. SIERPIŃSKI, Sur les suites infinies de fonctions définies dans les ensembles quelconques, Fund. Math. 24 (1935), 209-212. 